\title{
Opto-Magnetic Method for Epstein - Barr Virus and Cytomegalovirus Detection in Blood Plasma Samples
}

\author{
M. Papić-Obradović ${ }^{a}$, D. Kojić ${ }^{b}$ And A.L. Matija ${ }^{b}$ \\ ${ }^{a}$ Clinic for Gynecology and Obstetrics "Narodni front", Kraljice Natalije 62, 11000 Belgrade, Serbia \\ ${ }^{b}$ Department for Biomedical Engineering, Faculty of Mechanical Engineering, University of Belgrade \\ Kraljice Marije 16, 11120 Belgrade 35, Serbia
}

\begin{abstract}
Motivated by characterization of paramagnetic materials $(\mathrm{Al}, \mathrm{Mn}$ and $\mathrm{Ti})$ and diamagnetic materials $(\mathrm{Cu}$, $\mathrm{C}$ and $\mathrm{Zn}$ ) by opto-magnetic method that is based on light-matter interaction using digital imaging, we present results of Epstein-Barr virus (EBV) and cytomegalovirus (CMV) detection in blood plasma. To investigate light-blood plasma interaction we use wavelength difference of diffuse white light and reflected polarized light in red and blue channels of digital images (opto-magnetic method). Digital images of samples are analyzed by spectral convolution algorithm for light-matter interaction analysis. Since opto-magnetic method can detect very small difference between normal and pathological tissue states it is advantageous in comparison with classical methods. Especially it is important for early detection of suspicious tissue states and detection of viral infection presence in plasma. We compared our results with results of standard biomedical test for EBV and CVM, as a reference, and found out for group of 40 samples significant correlation of $93.6 \%$.
\end{abstract}

PACS numbers: 33.20.Kf, 33.57.+c, 42.25.Ja, 42.30.-d, 42.70.Jk, 87.19.xd

\section{Introduction}

Biomolecules and tissues are usually paramagnetic or diamagnetic materials, except blood where iron is present in erythrocytes (red blood cells). We investigate the presence of viral infection in blood plasma, which does not contain erythrocytes, and which is not ferromagnetic. The most common viruses that infect over $90 \%$ of world population are the Epstein-Barr virus (EBV) and cytomegalovirus (CMV), belonging to the herpes virus family. The manifestations of this infections are various, from persistent latent asymptomatic infection in immunocompetent population to development of wide spectrum of clinical manifestations that range from transient infections to aggressive malignancies in some immunocompromised patients [1, 2]. EBV is associated with etio-pathogenesis of an increasing number of cancers [3, 4].

Standard diagnostic methods related to EBV/CMV and their related diseases encompass serological methods (heterophile antibodies, immunofluorescence assays) [5], assays for cytotoxic T-cells and molecular diagnostic methods (in-situ hybridization, Southern blotting and polymerase chain reaction (PCR)) [2-6]. Methods that employ the interaction between matter and electromagnetic radiation are scarcely or not investigated at all. The introductory work in spectroscopy application to virology diagnostics [7] or possibilities of scanning electron microscopy [8] is present for some time but no significant studies have been undertaken so far. These methods were very successful in basic physics and chemistry research, but so far showed no perspective for clinical virology application.

And while most novel developments in molecular diagnostics are directed towards PCR based methods $[9,10]$, we explore a possibilities for introducing the application of a novel method that is based on opto-magnetic properties of light-matter interaction. Similar methodology uses method that is an inverse of opto-magnetism, or magneto-optical method, and has examined percentage of iron in an alloy [11].

\section{Materials and methods}

Our investigation of viral infection was performed over a sample set taken from 40 pregnant women that were classified by blood test in two groups: Epstein-Barr virus (EBV) group (32 cases, tests for presence of monoclonal antibodies, type IgM (M) and IgG (GM)) and Cytomegalovirus (CMV) group (8 cases M, GM). Each group was separated in two categories, positive (virus present, $16 \mathrm{EBV}$ and $4 \mathrm{CMV}$ ) and negative (virus absent, $16 \mathrm{EBV}$ and $4 \mathrm{CMV}$ ).

Testing on EBV and CMV was done in the Institute for Application of Nuclear Energy, Belgrade, Serbia (INEP). Diagnostic on EBV and CMV was done using standard enzyme immunoassay method (ELISA) $[12,13]$. Preparation of digital pictures for opto-magnetic finger print (OMF) was made by usage of dermoscopic imaging device (MySkin, USA) that has previously been successfully used in biophysical skin characterization (skin photo type, moisture, conductivity, etc.) [14].

After testing was performed in the INEP, samples were transferred to Biomedical Engineering Laboratory at the University of Belgrade, where comparison testing was performed by novel OMF method. The OMF technique is based on electron properties of matter (covalent bonds, hydrogen bonds, ion-electron interaction, van der Waals interaction) and its interaction with light 
$[15,16]$. Bearing in mind that the orbital velocity of valence electron in atoms is about $10^{6} \mathrm{~m} / \mathrm{s}$, this gives the ratio between magnetic force $\left(F_{\mathrm{M}}\right)$ and electrical force $\left(F_{\mathrm{E}}\right)$ of matter, of $F_{\mathrm{M}} / F_{\mathrm{E}} \approx 10^{-4}$. Since force $(F)$ is directly related to quantum action (Planck action, $h=F \times d \times t=6.626 \times 10^{-34} \mathrm{Js}$, where $d$ is displacement and $t$ is time of action) this means that the action of magnetic forces is four orders of magnitude closer to quantum action than the electrical ones. Since quantum state of matter is primarily responsible for conformational changes on the molecular level, this means that detecting differences between tissue states is by far more likely to give greater sensitivity on the level of magnetic forces than it would be on the level of measurement of electrical forces. Picture of surface that is taken by classical optical microscope is based on electromagnetic property of light, while the OMF is based on difference between diffuse white light (like that of daily light) and reflected polarized light. Reflected polarized light is produced when source of diffuse light irradiates the surface of matter under certain angle (Brewster's angle). Each type of matter has special different angle value of light polarization. We found out that angle of reflected polarized light of blood plasma is about $52^{\circ} \pm 0.8^{\circ}$. Since electrical component of reflected polarized light actively participates in light-matter interaction, then taking the difference between white light (both electrical and magnetic components of light are active in interaction with matter) and reflected polarized light (only electrical components of light is active in interaction with matter) yields magnetic properties of matter based on light-matter interaction.

We used digital images in RGB (R-red, G-green, B-blue) system in our analysis, therefore we chose basic pixel data in red and blue channels for white diffuse light $(\mathrm{W})$ and reflected polarized white light $(\mathrm{P})$. Algorithm for data analysis is based on chromaticity diagram called Maxwell's triangle [17], and spectral convolution operation according to ratio of (R-B)\&(W-P). The abbreviated designation means that the blue channel of the image is subtracted from the red channel (both belonging to white light) and the same procedure is applied to image obtained by reflected polarized light. Finally, both differences are used in spectral convolution algorithm to calculate data for opto-magnetic fingerprint of matter. Therefore, method and algorithm for creating unique spectral fingerprint are based on the convolution of RGB color channel spectral plots generated from digital images that capture single and multi-wavelength light-matter interaction for different paramagnetic materials (Al, Mn and $\mathrm{Ti}$ ), diamagnetic materials $(\mathrm{Cu}, \mathrm{C}$ and $\mathrm{Zn})$, alloys $\left(\mathrm{Pb}_{1-x} \mathrm{Mn}_{x} \mathrm{Te}\right)$ and biological tissues as paramagnetic/ diamagnetic materials (skin, biological water, amniotic fluid) [16].

Incident white light can give different information about properties of thin layer of matter (sample surface) depending on the angle of light incidence. When the incident white light is diffuse, the reflected white light is then composed of electrical and magnetic components (which are both longitudinal in this case), whereas diffuse incident light that is inclined under certain angle will produce reflected light, whose longitudinal wave contains only electrical component of light. However, magnetic component of light, as a transversal component of light wave, does not influence the CCD/CMOS camera sensor, were photon-to-electron conversion occurs.

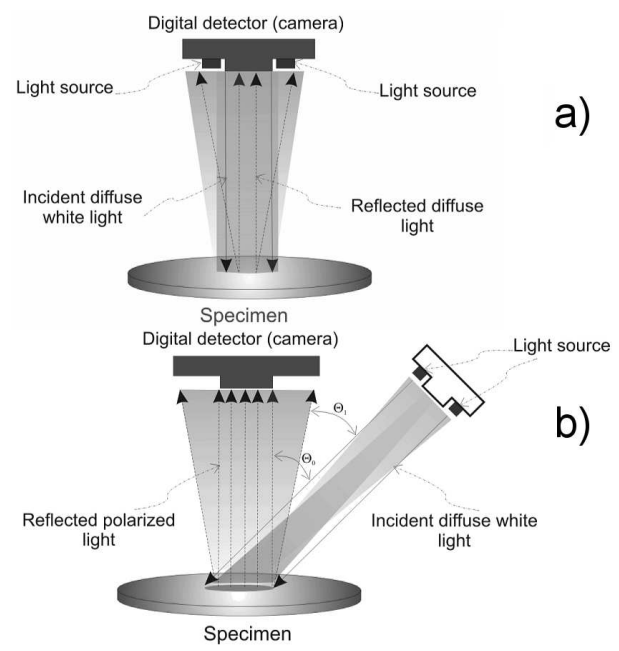

Fig. 1. The experimental arrangement sketch showing relative positions of light sources for white (a) and reflected polarized light (b). The degree of light polarization is $95.4 \%$, while and angular diffusion of the light source (six white LEDs arranged on circle) is $\pm 1.6^{\circ}$ (difference between angles $\theta$ and $\theta_{1}$ ).

\section{Results}

Typical digital images of samples tested positive for Epstein-Barr virus (EBV) and Cytomegalovirus (CMV) were taken with diffuse white light and reflected polarized light, respectively. Observation of images by naked eye would probably testify that there are no differences between them. However, using computer assisted analysis (CAA) based on pixel by pixel count and spectral convolution algorithm (SCA) [16], we find out significant difference whose final result is given in corresponding diagrams (Figs. 2-5).

Here we present only four typical cases of EBV (two $\operatorname{IgM}$ and two $\operatorname{IgG}$ ) to show difference between positive and negative of same cases (IgM or $\operatorname{IgG}$ ) and similarity of spectral data in DI-OMF of them.

\section{Discussion}

EVB is discovered in 1964 [4], its genome was sequenced in 1984 [18] and it has been recognized as the first human virus to be directly implicated in cancer genesis [3]. From this we see that the publications on this subject are mainly dedicated to better understanding of EVB biology and development of novel therapies 


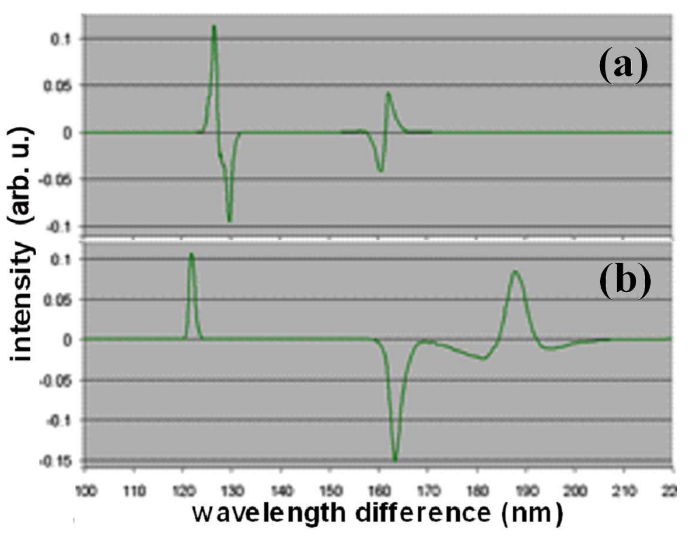

Fig. 2. Typical spectral data are based on (R-B)\&(W$\mathrm{P})$ spectral convolution obtained for two different samples that were tested positive (a) and negative (b) for presence of Epstein-Barr virus (Case 1: EBV-IgM). We observe four intensity peaks for positive and three for negative test results, with the following values for wavelength difference/intensity: (a) $126.6 \mathrm{~nm} / 0.113$, $129.7 \mathrm{~nm} /-0.095,160.8 \mathrm{~nm} /-0.041,162.1 \mathrm{~nm} / 0.041$, (b) $122.0 \mathrm{~nm} / 0.107,163.4 \mathrm{~nm} /-0.151,187.8 \mathrm{~nm} / 0.084$. Positive values designate the intensity of paramagnetic behavior while negative values designate the intensity of diamagnetic behavior; all values are comparative to standardized unit signal.

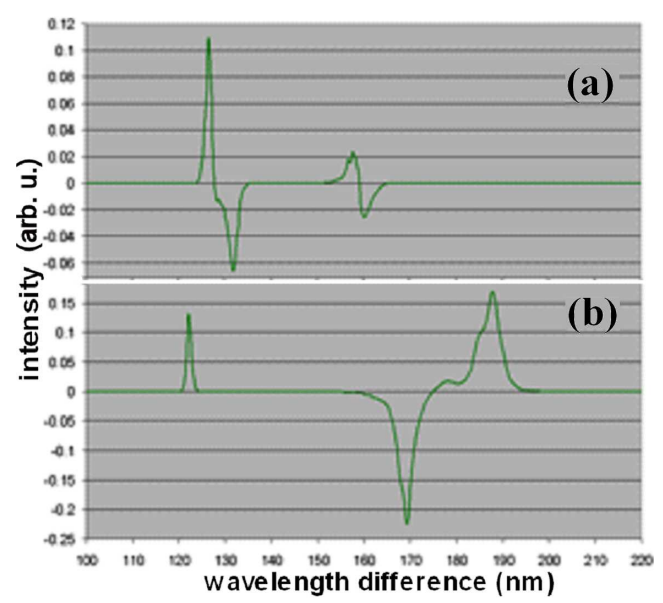

Fig. 3. Typical spectral data are based on (R-B)\&(W$\mathrm{P})$ spectral convolution obtained for two different samples that were tested positive (a) and negative (b) for presence of Epstein-Barr virus (Case 2: EBV-IgM). We observe four intensity peaks for positive and three for negative test results, with the following values for wavelength difference/intensity: (a) $126.6 \mathrm{~nm} / 0.110$, $132.3 \mathrm{~nm} /-0.060,157.8 \mathrm{~nm} / 0.023,160.2 \mathrm{~nm} /-0.026$, (b) $122.2 \mathrm{~nm} / 0.132,169.3 \mathrm{~nm} /-0.225,187.8 \mathrm{~nm} / 0.169$. Positive values designate the intensity of paramagnetic behavior while negative values designate the intensity of diamagnetic behavior; all values are comparative to standardized unit signal.

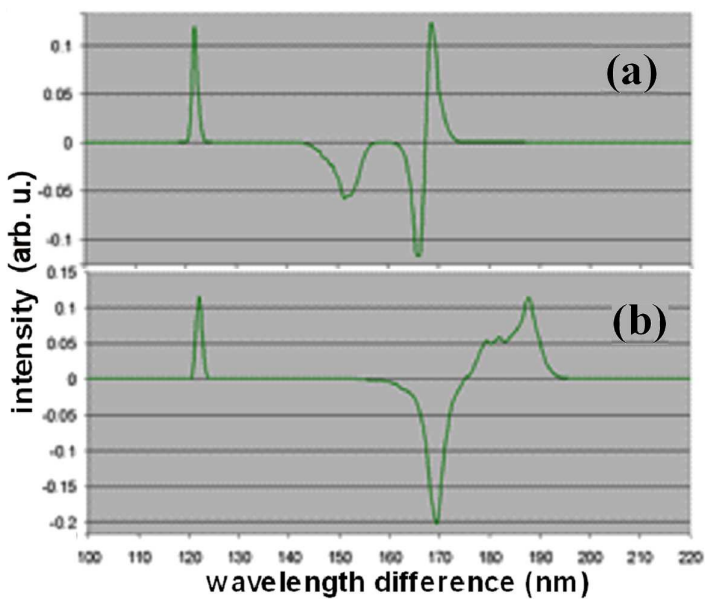

Fig. 4. Typical spectral data are based on (R-B)\&(WP) spectral convolution obtained for two different samples that were tested positive (a) and negative (b) for presence of Epstein-Barr virus (Case 3: EBV-IgG). We observe four intensity peaks for positive and three for negative test results, with the following values for wavelength difference/intensity: (a) $121.7 \mathrm{~nm} / 0.120$, $151.3 \mathrm{~nm} /-0.059,166.3 \mathrm{~nm} /-0.117,168.4 \mathrm{~nm} / 0.121$, (b) $122.0 \mathrm{~nm} / 0.115,169.3 \mathrm{~nm} /-0.203,187.8 \mathrm{~nm} / 0.114$. Positive values designate the intensity of paramagnetic behavior while negative values designate the intensity of diamagnetic behavior; all values are comparative to standardized unit signal.

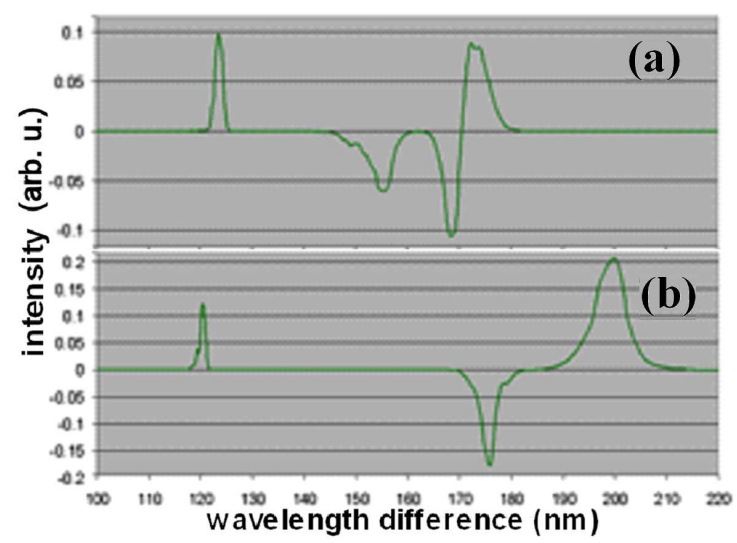

Fig. 5. Typical spectral data are based on (R-B)\&(W$\mathrm{P})$ spectral convolution obtained for two different samples that were tested positive (a) and negative (b) for presence of Epstein-Barr virus (Case 4: EBV-IgG). We observe four intensity peaks for positive and three for negative test results, with the following values for wavelength difference/intensity: (a) $123.6 \mathrm{~nm} / 0.098$, $155.7 \mathrm{~nm} /-0.061,168.4 \mathrm{~nm} /-0.106,172.2 \mathrm{~nm} / 0.087$, (b) $120.5 \mathrm{~nm} / 0.123,176.1 \mathrm{~nm} /-0.175,200.3 \mathrm{~nm} / 0.203$. Positive values designate the intensity of paramagnetic behavior while negative values designate the intensity of diamagnetic behavior; all values are comparative to standardized unit signal. 
for treating virus-associated diseases. However, EVB and CMV detection still remains within the scope of standard diagnostic methods $[2,5,6]$. Using opto-magnetic fingerprint method we developed an initial novel simple, fast, economical and accurate screening method for EVB and CMV detection using blood plasma.

Regarding the 40 samples examined for presence of EBV or CMV, two distinctive features can be observed in the given diagrams: number of peaks, position of peaks, distribution of peaks (up and down), and individual peak intensity. Regarding all features we see that it is possible to group these diagrams with respect to antibody type (IgG/IgM) and test result (positive/negative). The intensities as well as wavelength differences for IgM antibodies differ from those for IgG antibodies. All positive samples are approximated by four peaks while negative ones are approximated by only three. This is a promising evidence for using this method as a fast, accurate and economically affordable screening tool. Another feature, visible in the group of negative samples (around $180 \mathrm{~nm}$ ), does not exhibit an easily observable shape or peak position and therefore is excluded from this analysis.

Spectral data of all 40 cases (here presented are four ones in Figs. 2-5) display very good information regarding the difference between normal (negative) and virus infected (positive) samples. Because Omf spectral diagrams for Epstein-Barr virus (EBV-GM) and Cytomegalovirus (CMV-GM) appear similar, this algorithm still needs to be refined in order to more clearly distinguish which type of virus infection is present. in this stage of application, Omf method could be used as an adjunct method in virus detection since it yields good results in quick identification of virus infection presence. It can save time and money when used in parallel with expensive biochemical analysis.

\section{Acknowledgments}

We acknowledge Institute for Application of $\mathrm{Nu}-$ clear Energy (Serbia) for collecting samples and MySkin (USA) for providing devices to make and analyzed digital imaging. This research has been partially funded by Ministry of Science and Technological Development of Republic of Serbia, through Project TR19056, and partially by resources of company MySkin, USA (Patent $\mathrm{PCT} / \mathrm{US} 2008 / 050438)$.

\section{References}

[1] M.L. Gulley, J. Mol. Diagn. 3, 1 (2001).

[2] S. Tsuchiya, Crit. Rev. Oncol. Hematol. 44, 227 (2002).

[3] M.P. Thompson, R. Kurzrock, Clin. Cancer. Res. 10, 803 (2004).

[4] S.L. Young, A.B. Rickinson, Cancer 4, 757 (2004).

[5] R.F. Ambinder, R.B. Mann, Am. J. Pathol. 145, 239 (1994).

[6] S.A.S. Staras, S.C. Dollard, K.W. Radford, W.D. Flanders, R.F. Pass, M.J. Cannon, Clin. Infect. Dis. 43, 1143 (2006).

[7] P.J. Lambert, A.G. Whitman, O.F. Dyson, S.M. Akula, Virology J 3, 51 (2006).

[8] H. Ong, V. Chandran, J. Clin. Virol. 34, 195 (2005).

[9] L.L. Decker, L.D. Klaman, D.A. Thorley-Lawson, J. Virol. 70, 3286 (1996).

[10] R. Hassan, L.R. White, C.G. Stefanoff, D.E. de Oliveira, F.E. Felisbino, C.E. Klumb, C.E. Bacchi, H.N. Seuanez, I.R. Zalcberg, Diagn. Pathol. 1, 17 (2006).

[11] C.C. Huang, C.H. Su, M.Y. Liao, C.S. Yeh, Phys. Chem. Chem. Phys. 116331 (2009).

[12] M. Bhavnani, R.B. McGucken, J. Craske, J. Clin. Pathol. 44, 351 (1991).

[13] B. Weber, M. Brunner, W. Preiser, H.W. Doerr, J. Virol. Methods. 57, 87 (1996).

[14] J. Bandic, Dj. Koruga, R. Mehendale, S. Marinkovich: System, device and method for dermal imaging, US Pat. App. No. PCT/US2008/050438, Publication No: WO/2008/086311, Publication Date: 2008-07-17.

[15] Dj. Koruga, A. Tomic, Z. Ratkaj, L. Matija, Mat. Sci. For. 518, 491 (2006).

[16] Dj. Koruga, A. Tomic: Method and algorithm for analysis of light-matter interaction based on spectral convolution, US Pat. App. No. 61/061, 852, 2008, PCT/US2009/030347, Publ. No: WO/2009/089292, Publ. Date: 2009-07-16.

[17] G. Saxby, Practical Holography, 3rd ed., IOP Publishing Ltd., CRC Press, UK, 2004, p. 255.

[18] R. Baer, A.T. Bankier, M.D. Biggin, P.L. Deininger, P.J. Farrell, T.J. Gibson, G. Hatfull, G.S. Hudson, S.C. Satchwell, C. Séguin, P.S. Tuffnell, B.G. Barrell, Nature 310, 207 (1984). 